\title{
Abbreviations
}

AC alternating current

ACEEE American Council for an Energy Efficient Economy

AI artificial intelligence

AV autonomous vehicle

BA balancing area

BAA balancing area authority

CCA Community Choice Aggregation

CCS carbon capture and storage

CCUS carbon capture, utilization, and storage

CSAT customer satisfaction

DC direct current

DDPP U.S. Deep Decarbonization Pathways Project

DER distributed energy resources

DG distributed generation

DLMP distribution locational marginal pricing

DOE U.S. Department of Energy

DR demand response

DSO Distribution System Operator

EAAS energy as a service

ED energy democracy

EE energy efficiency

EIA U.S. Energy Information Administration

EIRP Energy Innovation Reform Project

ESCO energy service company

ESU Energy Service Utility 
EV electric vehicle

FERC Federal Energy Regulatory Commission

GDP gross domestic product

GHG greenhouse gas

GMP Green Mountain Power

GND Green New Deal

HVDC high-voltage direct current

ICT information and communications technology

IEA International Energy Agency

IOT Internet of Things

IOU investor-owned electric distribution utilities

IPCC Intergovernmental Panel on Climate Change

IPP independent power producer

ISO independent system operator

KPI key performance indicator

kWh kilowatt hour

LBNL Lawrence Berkeley National Labs

MCS Mid-Century Strategy

NCA U.S. National Climate Assessment

NEEP Northeast Energy Efficiency Partnership

NOAA National Oceanic and Atmospheric Administration

NREL National Renewable Energy Lab

NWA non-wires alternative

PBR performance based regulation

POLR Provider of Last Resort

PPA power purchase agreement

PQR power quality and reliability

$\mathrm{PSH} \quad$ pumped storage hydro

PTG power-to-gas

P2G2P power-to-gas-to-power

PV photovoltaic solar

PwC PricewaterhouseCoopers

R\&D research and development

RIIO Revenue $=$ Incentives + Innovation + Outputs

RMI Rocky Mountain Institute

RPS renewable portfolio standards

RTO regional transmission operator 
SECC Smart Energy Consumer Collaborative

SI Smart Integrator

SMR small modular nuclear reactor

STEM science, technology, engineering, and mathematics

TO transmission operator

TOD time-of-day pricing

TSO transmission system operator

UNFCCC United Nations Framework Convention on Climate Change

VRE variable renewable energy 



\section{POWER AFTER CARBON}


